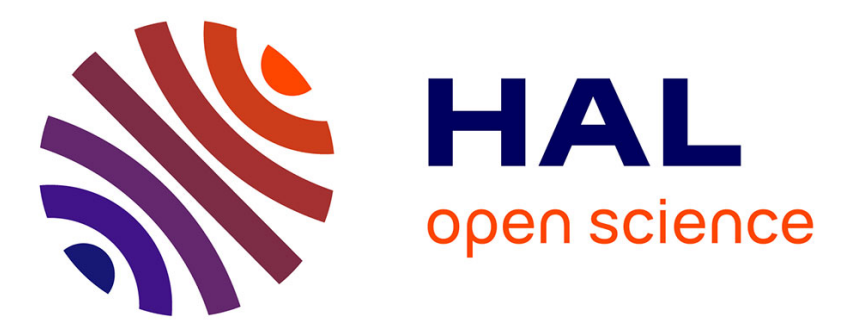

\title{
Sensor noise measurement in the presence of a flickering illumination
}

Frédéric Sur, Michel Grediac

\section{To cite this version:}

Frédéric Sur, Michel Grediac. Sensor noise measurement in the presence of a flickering illumination. ICIP - IEEE International Conference on Image Processing, Oct 2014, Paris, France. p. 1763-1767, 10.1109/ICIP.2014.7025353 . hal-01022379

\section{HAL Id: hal-01022379 \\ https://inria.hal.science/hal-01022379}

Submitted on 10 Jul 2014

HAL is a multi-disciplinary open access archive for the deposit and dissemination of scientific research documents, whether they are published or not. The documents may come from teaching and research institutions in France or abroad, or from public or private research centers.
L'archive ouverte pluridisciplinaire $\mathbf{H A L}$, est destinée au dépôt et à la diffusion de documents scientifiques de niveau recherche, publiés ou non, émanant des établissements d'enseignement et de recherche français ou étrangers, des laboratoires publics ou privés. 


\section{SENSOR NOISE MEASUREMENT IN THE PRESENCE OF A FLICKERING ILLUMINATION}

\author{
Frédéric SUR \\ LORIA UMR 7503 \\ CNRS - INRIA - Université de Lorraine (France)
}

\author{
Michel GRÉDIAC \\ Institut Pascal UMR 6602 \\ CNRS - Université Blaise Pascal (France)
}

\begin{abstract}
Raw data from a digital imaging sensor are impaired by a heteroscedastic noise, the variance of pixel intensity linearly depending on the expected value. The most natural way of estimating the variance and the expected value at a given pixel is certainly empirical estimation from the variations along a stack of images of any static scene acquired at different times under the same camera setting. However, the relation found between the sample variance and the sample expectation is actually not linear, especially in the presence of a flickering illumination. The contribution of this paper is twofold. First, a theoretical model of this phenomenon shows that the linear relation changes into a quadratic one. Second, an algorithm is designed, which not only gives the parameters of the expected linear relation, but also the whole set of parameters governing an image formation, namely the gain, the offset and the readout noise. The rolling shutter effect is also considered.
\end{abstract}

Index Terms - Noise measurement, raw image, PoissonGaussian noise, flickering illumination, rolling shutter.

\section{INTRODUCTION}

The raw output of a linear camera (either CCD or CMOS) is basically proportional to the quantity of light photons arriving at a sensor cell, plus a dark current and readout noise. The following stochastic model for the raw output of a linear imaging sensor is often used in the literature $[1,2,3,4]$

$$
u(x, y)=g \eta_{p(x, y)+d(x, y)}(x, y)+\delta(x, y)
$$

- $u(x, y)$ is the intensity (gray level) measured at the photosite corresponding to the pixel $(x, y)$ in the output raw image. - $g>0$ is the gain of the electronic system.

- The number of generated electrons $\eta_{p(x, y)+d(x, y)}$ at $(x, y)$ is a random variable following a Poisson distribution of mean $p(x, y)+d(x, y)$, assumed to be spatially independently distributed. This gives the shot noise. Here $p$ is the number of collected photo-electrons and $d$ is the number of spontaneously generated dark electrons. With short exposures, dark current is negligible and we set in this paper $d=0$.

- $\delta(x, y)$ is a Gaussian white noise of mean $\mu$ (fixed offset value imposed by the manufacturer) and variance $\sigma^{2}$ (due to readout and quantization noise).

- $\eta_{p(x, y)}$ and $\delta$ are independent random variables.
A Poisson variable having equal expectation and variance,

$$
\left\{\begin{array}{l}
E(u(x, y))=g p(x, y)+\mu \\
\operatorname{Var}(u(x, y))=g^{2} p(x, y)+\sigma^{2}
\end{array}\right.
$$

where $E$ denotes the expectation and Var the variance of any random variable. Consequently, a linear relation holds:

$$
\operatorname{Var}(u(x, y))=g E(u(x, y))+\sigma^{2}-g \mu
$$

Estimating the whole set of noise parameters can be achieved by the so-called "photon transfer method" [5] which needs a controlled experimental setting. However, estimating the slope $g$ and the intercept $\sigma^{2}-g \mu$ in (3) is sufficient for many applications, e.g., for stabilizing the noise variance $[2,6]$. In this case, the authors of $[2,4,7,8,9]$ propose algorithms to estimate the slope and the intercept based on linear regression and a single image. Sample mean and variance are evaluated on image regions from a preliminary segmentation which is likely to fail in the case of a cluttered scene.

Instead of using spatial statistics of a single image, it is possible to use temporal statistics from a series of images. Such an approach simply consists in taking a series of images of a static scene at different times, and in computing afterwards the sample mean and variance at a given pixel as in [10]. However, it turns out that the random fluctuations of the stacked pixel intensities are not solely the result of the digital noise modeled by (1), as illustrated in Figure 1. An important external source of intensity fluctuations is indeed a non-constant illumination of the sensor. Two main reasons cause this phenomenon: a flickering illumination (certain light sources such as neon tubes are particularly affected by the domestic alternative current), or equivalently a slight variability of the exposure caused by mechanical or electronic imperfections of the camera shutter or lens diaphragm. In the context of denoising from a burst of images, the authors of [11] found out that the bursts actually suffer from changes in the light intensity, which they circumvent by histogram equalization [12]. Although this method is effective for denoising, it does not enable accurate noise parameter measurement.

A theoretical model is proposed in Section 2 and a practical algorithm to estimate the noise parameters from a series of images under a flickering illumination is discussed in Section 3. The rolling shutter effect is shortly discussed in Section 4. Section 5 demonstrates that light flickering makes it possible to estimate each of the sensor parameters $(g, \sigma, \mu)$. 

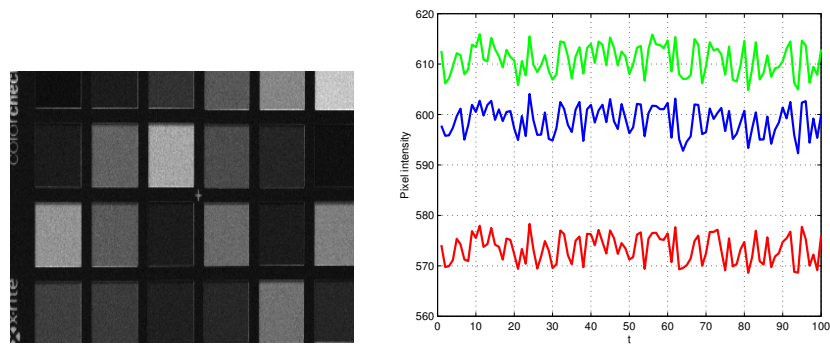

Fig. 1. A burst of 100 images of a X-Rite colorchecker (left) is taken, lit by a neon light under constant camera parameters. The burst frequency is not constant. An average intensity is computed over 100 pixels belonging to the same color patch in order to discard the random noise. The graph (right) depicts the evolution of the average calculated from three different patches. In-phase fluctuations are caused by light flickering.

\section{PIXEL INTENSITY AND FLICKERING LIGHT}

We consider a series of $T$ images of any static scene, aperture and shutter speed setting being constant. The number of emitted electrons being proportional to the number of incoming photons (through the quantum efficiency), the average number of electrons at a photosite of the $t$-th image is $\left(1+\gamma_{t}\right) p$, where $1+\gamma_{t}$ is the relative variation of the light intensity. From (1), the intensity at pixel $(x, y)$ of the $t$-th image is

$$
u(x, y, t)=g \eta_{\left(1+\gamma_{t}\right) p(x, y)}(x, y, t)+\delta(x, y, t)
$$

where $\eta$ and $\delta$ are as in (1), and $\gamma_{t}$ is an identically distributed 0 -mean random process. The variance of $\gamma$ is noted $\sigma_{\gamma}^{2}$.

In the following calculations, we make use of the law of the total expectation and the law of the total variance: if $X$ and $Y$ are two random variables, then $E(X)=E(E(X \mid Y))$ and $\operatorname{Var}(X)=E(\operatorname{Var}(X \mid Y))+\operatorname{Var}(E(X \mid Y))$. We note $\bar{X}$ the sample mean $1 / T \sum_{t=1}^{T} X(t)$ of any random process $(X(t))_{1 \leq t \leq T}$. Under independence assumption, the variance of $\bar{X}$ is $1 / T^{2} \sum_{t=1}^{T} \operatorname{Var}(X(t))$.

Let us skip for a while $(x, y, t)$ after $u, \delta$, or $p$. First,

$$
\begin{aligned}
E(u) & =E\left(E\left(g \cdot \eta_{\left(1+\gamma_{t}\right) p}+\delta \mid \gamma_{t}\right)\right) \\
& =E\left(g\left(1+\gamma_{t}\right) p\right)+\mu \\
& =g p+\mu
\end{aligned}
$$

The temporal sample mean $\bar{u}(x, y)$ is hence an unbiased estimation of $E(u(x, y, t))=g p(x, y)+\mu$. Second,

$$
\begin{aligned}
\operatorname{Var}(u)= & E\left(\operatorname{Var}\left(g \cdot \eta_{\left(1+\gamma_{t}\right) p}+\delta \mid \gamma_{t}\right)\right) \\
& \left.+\operatorname{Var}\left(E\left(g \cdot \eta_{\left(1+\gamma_{t}\right) p}+\delta \mid \gamma_{t}\right)\right)\right) \\
= & E\left(g^{2}\left(1+\gamma_{t}\right) p\right)+\sigma^{2} \\
& +\operatorname{Var}\left(g\left(1+\gamma_{t}\right) p+\mu\right) \\
= & g^{2} p+\sigma^{2}+g^{2} p^{2} \sigma_{\gamma}^{2}
\end{aligned}
$$

Hence $\operatorname{Var}(u)$ does not depend on $t$. In particular, $\operatorname{Var}(\bar{u})=$ $\operatorname{Var}(u) / T$. Moreover, since $g p=E(u)-\mu$,

$$
\begin{aligned}
& \operatorname{Var}(u(x, y, t))=\sigma_{\gamma}^{2} E^{2}(u(x, y, t)) \\
&+\left(g-2 \sigma_{\gamma}^{2} \mu\right) E(u(x, y, t))+\sigma^{2}-g \mu+\sigma_{\gamma}^{2} \mu^{2}
\end{aligned}
$$

Consequently, the light flickering changes the linear relation of (3) into a quadratic relation. Interestingly, [13] (cited by [14]) shows that such a relation also holds for intensified charged couple device (ICCD), with a different model.

It turns out that a part of non-random intensity fluctuations can also be explained by vibrations of the camera, which affect the intensity of pixels along edges, as discussed in [15, 16]. These pixels (as well as clipped data) are simply discarded in a preliminary step.

\section{ESTIMATING NOISE PARAMETERS}

The proposed algorithm aims at estimating the noise parameters. It consists in the following steps:

1. Discard pixels with large intensity gradient in order to only keep pixels not affected by vibrations;

2. Identify the flicker parameter $\gamma_{t}$ for each image by a nonlocal method, and deduce a linear flicker-free variance;

3. Estimate the noise parameters with linear regression.

The output of the proposed algorithm is an estimation of every parameter of the linear camera model, namely $g, \mu$, and $\sigma$, and not solely of the parameter $g$ and $\sigma^{2}-g \mu$ of the linear relation (3) as in [2, 4, 7, 8, 9].

1. Discarding pixels affected by physical vibrations. We only keep pixels where the intensity gradient is likely to be caused by random noise instead of edge pixels whose intensity fluctuates because of mechanical vibrations. Gradient being evaluated with a centered difference scheme, one has $\|\nabla \bar{u}\|^{2}=(\bar{u}(x+1, y)-\bar{u}(x-1, y))^{2} / 4+(\bar{u}(x, y+1)-$ $\bar{u}(x, y-1))^{2} / 4$. Assuming that $E(u)$ is constant in the 4neighbourhood of $(x, y)$ and that pixel intensities are independently distributed, $(\bar{u}(x+1, y)-\bar{u}(x-1, y)) / 2$ has 0 -mean and variance $\operatorname{Var}(u(x, y, t)) /(2 T)$. Hence, under Gaussian assumption, $2 T\|\nabla \bar{u}\|^{2} / \operatorname{Var}(u(x, y, t))$ follows a $\chi^{2}$ law with 2 degrees of freedom. We discard pixels such that the p-value of $2 T\|\nabla \bar{u}\|^{2} / \bar{\sigma}_{u}^{2}$ is below $1 \%$, with $\bar{\sigma}_{u}^{2}$ the sample variance.

2. Removing flicker and estimating $\mu$ and $\left(\gamma_{\mathbf{t}}\right)_{\mathbf{1} \leq \mathbf{t} \leq \mathbf{T} \text {. }}$ One has $E\left(u \mid \gamma_{t}\right)=g\left(1+\gamma_{t}\right) p+\mu$ with unknown $g$ and $\mu$. The difficulty to estimate $\gamma_{t}$ is that it needs an estimation of the expectation for any image of the stack, and also of the expected photo-electron count $p$. Since $E(\bar{u})=g p+\mu$, we form the ratio:

$$
v(x, y, t)=\frac{u(x, y, t)}{\bar{u}(x, y)}
$$

If $X$ and $Y$ are random variables, a Taylor expansion around $E(X), E(Y)$ gives the approximation:

$$
\left\{\begin{array}{l}
E\left(\frac{X}{Y}\right) \simeq \frac{E(X)}{E(Y)}-\frac{\operatorname{Cov}(X, Y)}{E(Y)^{2}}+\frac{E(X)}{E(Y)^{3}} \operatorname{Var}(Y) \\
\operatorname{Var}\left(\frac{X}{Y}\right) \simeq \frac{\operatorname{Var}(X)}{E(Y)^{2}}-\frac{2 E(X)}{E(Y)^{3}} \operatorname{Cov}(X, Y)+\frac{E(X)^{2}}{E(Y)^{4}} \operatorname{Var}(Y)
\end{array}\right.
$$

Hence

$$
\left\{\begin{array}{l}
E\left(v(x, y, t) \mid \gamma_{t}\right)=\frac{g\left(1+\gamma_{t}\right) p(x, y)+\mu}{g p(x, y)+\mu}+\mathcal{O}\left(\frac{1}{T}\right) \\
\operatorname{Var}\left(v(x, y, t) \mid \gamma_{t}\right)=\frac{g\left(1+\gamma_{t}\right) E(u(x, y, t))+\sigma^{2}-g\left(1+\gamma_{t}\right) \mu}{E(\bar{u}(x, y))^{2}}+\mathcal{O}\left(\frac{1}{T}\right)
\end{array}\right.
$$


The variance of $v$ is minimum for large values of $E(\bar{u}(x, y))$. Let us define the set $\mathcal{S}^{*}=\left\{(x, y), u^{*}-2 \sigma_{u^{*}} \leq \bar{u}(x, y) \leq\right.$ $\left.u^{*}+2 \sigma_{u^{*}}\right\}$ where $u^{*}$ is the $99 \%$ quantile in the intensity distribution of the averaged image $\bar{u}=g p+\mu+\mathcal{O}(1 / T)$, and $\sigma_{u^{*}}$ is the associated standard deviation. $g p+\mu$ is assumed constant across $\mathcal{S}^{*}$, equal to $u^{*}$. Consequently, an estimation of $E\left(v(x, y, t) \mid \gamma_{t}\right)$ for $g p(x, y)=u^{*}-\mu$ is given by a sample mean over the set $\mathcal{S}^{*}$. Indeed, from (14), for any $t$, $v^{*}(t)=\left(\sum_{(x, y) \in \mathcal{S}^{*}} v(x, y, t)\right) / \# \mathcal{S}^{*}$ is a random variable of expectation and variance

$$
\left\{\begin{array}{l}
E\left(v^{*}(t)\right)=\frac{1+\gamma_{t}+\mu /\left(u^{*}-\mu\right)}{1+\mu /\left(u^{*}-\mu\right)}+\mathcal{O}\left(\frac{1}{T}\right) \\
\operatorname{Var}\left(v^{*}(t)\right)=\mathcal{O}\left(\frac{1}{\# \mathcal{S}}+\frac{1}{T \# \mathcal{S}}\right)
\end{array}\right.
$$

where $\# \mathcal{S}^{*}$ denotes the cardinality of $\mathcal{S}^{*}$.

Once $v^{*}(t)$ has been calculated, $\widetilde{u}$ defined for any $(x, y, t)$ by $\widetilde{u}(x, y, t)=u(x, y, t)-v^{*}(t) \bar{u}(x, y)$ is such that:

$$
\left\{\begin{aligned}
& E\left(\widetilde{u}(x, y, t) \mid \gamma_{t}\right)=g\left(1+\gamma_{t}\right) p(x, y)+\mu \\
&-\left(\frac{1+\gamma_{t}+\mu /\left(u^{*}-\mu\right)}{1+\mu /\left(u^{*}-\mu\right)}+\mathcal{O}\left(\frac{1}{T}\right)\right)(g p(x, y)+\mu) \\
&=-\gamma_{t} \mu\left(\frac{u^{*}-\mu}{u^{*}}-\frac{g p(x, y)}{u^{*}}\right)+\mathcal{O}\left(\frac{1}{T}\right) \\
& \operatorname{Var}\left(\widetilde{u}(x, y, t) \mid \gamma_{t}\right)=g^{2}\left(1+\gamma_{t}\right) p(x, y)+\sigma^{2}+\mathcal{O}\left(\frac{1}{T}\right)
\end{aligned}\right.
$$

From now on, we assume that the $\mathcal{O}(1 / T)$ terms can be neglected (i.e., the series is made of a sufficient number $T$ of images).

Let $K=E\left(\widetilde{u}(x, y, t) \mid \gamma_{t}\right) /\left(E\left(v^{*}(t)\right)-1\right)$. One has

$$
K=-\mu\left(\frac{u^{*}-\mu}{u^{*}}-\frac{g p}{u^{*}}\right) \frac{u^{*}}{u^{*}-\mu}=-\mu\left(1-\frac{g p}{u^{*}-\mu}\right)
$$

We can see that $K$ does not depend on $t$. For a fixed $p(x, y)$, there is the same proportionality between $E\left(\widetilde{u}(x, y, t) \mid \gamma_{t}\right)$ and $E\left(v^{*}(t)-1\right)$ for every $t$. The proportionality coefficient $K$ can be estimated by linear regression. Similarly to the estimation of $E\left(v^{*}(t)\right)$, we choose an iso-value set to estimate, for every $t, E\left(\widetilde{u}(x, y, t) \mid \gamma_{t}\right)$. From (16), the smallest value of $\operatorname{Var}\left(\widetilde{u}(x, y, t) \mid \gamma_{t}\right)$ is attained for small $p(x, y)$. We thus define the set $\mathcal{S}_{*}=\left\{(x, y), u_{*}-2 \sigma_{u_{*}} \leq \bar{u}(x, y) \leq u_{*}+2 \sigma_{u_{*}}\right\}$ where $u_{*}$ is the $1 \%$ quantile in the intensity distribution of the averaged image $\bar{u}(x, y)$ and $\sigma_{u_{*}}$ the associated standard deviation. Similar to $v^{*}$, an estimator of $E\left(\widetilde{u}(x, y, t) \mid \gamma_{t}\right)$ for $g p(x, y)=u_{*}-\mu$ is $v_{*}(t)=\left(\sum_{(x, y) \in \mathcal{S}_{*}} \widetilde{u}(x, y, t)\right) / \# \mathcal{S}_{*}$.

Consequently, a linear regression on the scatter plot $\left(v_{*}(t), v^{*}(t)-1\right)$ gives an estimation of $K$.

Moreover, from (17),

$$
K=-\mu\left(1-\frac{u_{*}-\mu}{u^{*}-\mu}\right)
$$

Note that $K<0$. Besides, $K\left(u^{*}-\mu\right)=-\mu\left(u^{*}-u_{*}\right)$ and:

$$
\mu=\frac{K u^{*}}{K-u^{*}+u_{*}}
$$

Once $\mu$ is computed from the estimated $K$, we deduce from (15) that an estimator $\widetilde{\gamma}_{t}$ of $\gamma_{t}$ is given for any $t$ by:

$$
\widetilde{\gamma}(t)=\left(v^{*}(t)-1\right)\left(1+\frac{\mu}{u^{*}-\mu}\right)
$$

With these estimations of $\gamma_{t}$ and $\mu$, it would be possible to obtain $g$ and $\sigma$ by a quadratic regression from (11). However, this proves to be inaccurate. Instead, we derive a variance estimation unaffected by flicker. Identifying the estimations of $\gamma_{t}$ and $\mu$ with their actual values, let us define:

$$
\widehat{u}(x, y, t)=u(x, y, t)-\left(1+\gamma_{t}\right)(\bar{u}(x, y)-\mu)
$$

This quantity is such that:

$$
\begin{aligned}
E(\widehat{u}(x, y, t))= & E\left(E\left(\widehat{u}(x, y, t) \mid \gamma_{t}\right)\right)=E(\mu)=\mu \\
\operatorname{Var}(\widehat{u}(x, y, t))= & E\left(\operatorname{Var}\left(\widehat{u}(x, y, t) \mid \gamma_{t}\right)\right) \\
& +\operatorname{Var}\left(E\left(\widehat{u}(x, y, t) \mid \gamma_{t}\right)\right) \\
= & E\left(g^{2}\left(1+\gamma_{t}\right) p(x, y)+\sigma^{2}\right. \\
& +\left(1+\gamma_{t}\right)^{2} \operatorname{Var}(\bar{u}(x, y))+\operatorname{Var}(\mu) \\
= & g^{2} p(x, y)+\sigma^{2}+\left(1+\sigma_{\gamma}^{2}\right) \operatorname{Var}(\bar{u}(x,(25) \\
= & g E(u(x, y, t))+\sigma^{2}-g \mu \\
& +\left(1+\sigma_{\gamma}^{2}\right) \operatorname{Var}(\bar{u}(x, y))
\end{aligned}
$$

Since $\operatorname{Var}(\bar{u}(x, y))=\mathcal{O}(1 / T)$, by neglecting this term as above, we can write the following linear relation between the variance of the corrected stack $\widehat{u}(x, y, t)$ and the expected value of $u(x, y, t)$ (which does not depend on the light flickering, cf. (7)):

$$
\operatorname{Var}(\widehat{u}(x, y, t))=g E(u(x, y, t))+\sigma^{2}-g \mu
$$

which is the same relation as in the standard model of (3).

In practice, we weight the sum in the definition of $v^{*}$ by $\left.e^{-\left(\bar{u}(x, y)-u^{*}\right)^{2} /\left(2 \sigma_{u^{*}}^{2}\right)}\right)$, and similarly for $v_{*}$. This acts as a soft threshold in the definition of $\mathcal{S}^{*}$ or $\mathcal{S}_{*}$ as noted in another context in [17]. By construction, $v^{*}(t)$ and $v_{*}(t)$ are non-local estimators [18].

3. Linear regression. A linear regression over the scatter plot of the sample variance of the $\widehat{u}(x, y, t)$ against the sample mean of the $u(x, y, t)$ gives us estimations of the slope $g$, and intercept $\sigma^{2}-g \mu$, hence an estimation of $\sigma$ since $\mu$ is known at this stage by (19).

\section{BALANCING THE ROLLING SHUTTER EFFECT}

In a digital single-lens reflex (DSLR) camera with a focalplane shutter, pixels are not lit simultaneously. This is all the more noticeable when fast shutter speeds are used, especially with speeds faster than X-sync. CMOS cameras with an electronic shutter are also affected. This does not change the quadratic relation of (11) which only involves the variance $\sigma_{\gamma}^{2}$ and is based on a local argument, but this makes ineffective the image-wide estimation of each $\gamma_{t}$ in step 2, Section 3.

The proposed solution is to perform the estimation of $\gamma_{t}$ on horizontal bands of a fixed width centered at row $y$ (since the DSLR of Section 5 have vertical-travel shutters), which gives a $\gamma_{t}(y)$ which is then plugged in (21). The width is taken here equal to 40 pixels for the fastest shutter speeds. 

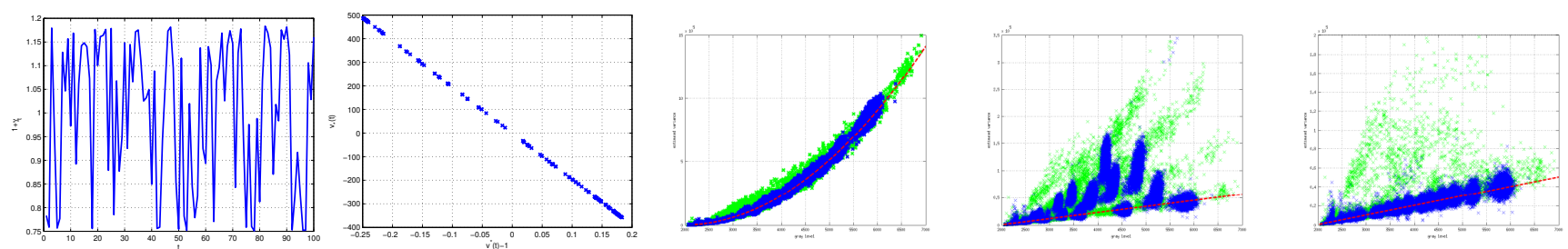

Fig. 2. Left to right: estimation of the light fluctuation $1+\gamma_{t} ; v_{*}(t)$ against $v^{*}(t)-1$; sample variance of $u(x, y, t)$ against sample mean of $u(x, y, t)$; sample variance of $\widehat{u}(x, y, t)$ against sample mean of $u(x, y, t)$ (image-wide estimation); the same statistics after local estimation (to balance rolling shutter effect).

\begin{tabular}{|c|c|c|c|c|c|c|c|c|c|c|c|c|c|c|}
\hline & & & \multicolumn{4}{|c|}{ 1. Photon transfer method ${ }^{1}$} & \multicolumn{2}{|c|}{ 2. Foi et $\mathrm{al}^{2}$} & \multicolumn{4}{|c|}{ 3. Proposed method } & \multicolumn{2}{|c|}{ 4. Linear estimation } \\
\hline & ISO & $t$ (sec.) & $g$ & $\sigma$ & $\mu$ & $\sigma^{2}-g \mu$ & $g$ & $\sigma^{2}-g \mu$ & $g$ & $\sigma$ & $\mu$ & $\sigma^{2}-g \mu$ & $g$ & $\sigma^{2}-g \mu$ \\
\hline \multirow{3}{*}{$\begin{array}{l}\text { EOS 350D } \\
\text { Colorch. }\end{array}$} & 100 & $1 / 15$ & 0.096 & 2.21 & 256 & -19.92 & 0.093 & -20.04 & 0.087 & 2.27 & 260.0 & -17.49 & 0.112 & -25.18 \\
\hline & 400 & $1 / 30$ & 0.384 & 2.88 & 256 & -91.92 & 0.310 & -69.60 & 0.358 & 2.53 & 256.8 & -85.55 & 0.406 & -100.7 \\
\hline & 1600 & $1 / 125$ & 1.531 & 6.36 & 256 & -355.3 & 1.472 & -342.1 & 1.455 & 4.62 & 254.8 & -349.4 & 3.741 & -963.7 \\
\hline \multirow{4}{*}{$\begin{array}{l}\text { EOS 6D } \\
\text { Colorch. }\end{array}$} & 100 & $1 / 3$ & 0.176 & 4.85 & 2047 & -338.6 & 0.548 & -1230.6 & 0.209 & 5.38 & 2158.4 & -422.2 & 0.269 & -573.4 \\
\hline & 400 & $1 / 15$ & 0.705 & 5.31 & 2047 & -1442 & 0.863 & -1769.8 & 0.697 & 3.16 & 2053.7 & -1421.2 & 0.876 & -1880.1 \\
\hline & 1600 & $1 / 80$ & 2.69 & 7.91 & 2047 & -5534 & 2.75 & -5545.9 & 2.57 & 6.85 & 2047.5 & -5222.5 & 6.22 & -13846 \\
\hline & 6400 & $1 / 320$ & 10.52 & 19.03 & 2047 & -21131 & 10.80 & -21755 & 10.49 & 16.25 & 2043.7 & -21170 & 122.0 & -281840 \\
\hline \multirow{3}{*}{$\begin{array}{l}\text { EOS 350D } \\
\text { Clutter }\end{array}$} & 100 & $1 / 13$ & 0.096 & 2.21 & 256 & -19.92 & 0.115 & -25.83 & 0.093 & 1.99 & 247.3 & -19.07 & 0.132 & -29.89 \\
\hline & 400 & $1 / 60$ & 0.384 & 2.88 & 256 & -91.92 & 0.446 & -107.3 & 0.375 & 3.51 & 258.9 & -84.81 & 0.730 & -186.4 \\
\hline & 1600 & $1 / 250$ & 1.531 & 6.36 & 256 & -355.3 & 1.481 & -343.8 & 1.503 & 6.60 & 258.7 & -345.2 & 4.64 & -1207.1 \\
\hline \multirow{4}{*}{$\begin{array}{l}\text { EOS 6D } \\
\text { Clutter }\end{array}$} & 100 & $1 / 2$ & 0.176 & 4.85 & 2047 & -338.6 & 0.249 & -504.2 & 0.202 & 5.36 & 2115.4 & -399.0 & 0.197 & -397.2 \\
\hline & 400 & $1 / 10$ & 0.705 & 5.31 & 2047 & -1442 & 0.825 & -1692.7 & 0.660 & 7.28 & 2094.3 & -1330 & 0.643 & -1294.7 \\
\hline & 1600 & $1 / 40$ & 2.69 & 7.91 & 2047 & -5534 & 2.79 & -5669.9 & 2.51 & 9.77 & 2058.7 & -5072.8 & 4.28 & -8959.1 \\
\hline & 6400 & $1 / 160$ & 10.52 & 19.03 & 2047 & -21131 & 10.78 & -21748 & 10.31 & 21.85 & 2058.2 & -20752 & 52.98 & -123430 \\
\hline
\end{tabular}

Table 1. Estimating noise parameters, comparison with existing methods (values in "gray-level" units as in [5]).

\section{EXPERIMENTS AND DISCUSSION}

Figure 2 shows an example. $T=100$ images of an X-Rite Colorchecker are taken with a Canon EOS 6D camera (row ISO 6400 in table 1, hence a fast shutter speed, prone to rolling shutter effect). The light flickering $1+\gamma_{t}$ is estimated and $\sigma_{\gamma}=0.15$ is found. The scatter plot of $v_{*}(t)$ against $v^{*}(t)-1$ shows the linear relation of slope $K<0$. The graph of the sample variance of $u(x, y, t)$ against the sample expectation has a quadratic trend as predicted by (11). The plot for the variance of $\widehat{u}(x, y, t)$ is not linear when $\gamma_{t}$ is assumed constant over the image: pixels belonging to some color patches are not adequately corrected, which explains the clusters out of the global trend. With an estimation performed as in Section 4, the trend becomes linear. The green points are the outliers discarded by Step 1 (pixels affected by vibrations), and the estimations are based on the blue points.

Table 1 gives the results of a comparison. Two Canon DSLR cameras (the old 12-bit EOS 350D and the recent 14bit EOS 6D) shoot two different scenes lit by a neon light: an $\mathrm{X}$-Rite Colorchecker and a cluttered scene (textured objects lying on a desk). Several ISO values are tested and the corresponding exposure time $t$ (depending on the aperture) are given. A series of 100 raw images is linearly processed with dcraw $^{3}$ and the red channel is extracted; other channels give

\footnotetext{
${ }^{1}$ EOS 350D: from www.astrosurf.com/comolli/strum41.htm EOS 6D: from www.astrosurf.com/comolli/strum54.htm

${ }^{2}$ v. 2.31 implementing [4], www.cs.tut.fi/ foi/sensornoise.html

${ }^{3}$ options: dcraw -C -D - 4 -r 1111
}

similar results. We give: (1) the values of the photon transfer method ${ }^{1}$ ( $\mu$ estimated independently); (2) the output of a software ${ }^{2}$ based on a single image (hence not affected by flickering) which estimates $g$ and $\sigma^{2}-g \mu ;(3)$ the results of the proposed method; (4) the estimation of the slope $g$ and the intercept $\sigma^{2}-g \mu$ of a regression line from $(E(u), \operatorname{Var}(u))$, disregarding the quadratic trend. Concerning $g$ and $\sigma^{2}-g \mu$, the colorchecker scene basically gives similar results for $\mathbf{1}$, 2, 3. However, the results of $\mathbf{2}$ are poorer (and even totally wrong in some cases) on the cluttered scene, which is more difficult to segment. In return, the proposed method requires a series of images. The over-estimation of $g$ by $\mathbf{4}$ shows that it is important to correct flickering. We can see that the proposed method not only correcly estimates $g$, but also $\sigma$ and $\mu$.

Concerning the exposure time, since the neon light is powered by $50 \mathrm{~Hz}$ current, its flickering should not be noticeable for low ISO values. However, $\sigma_{\gamma}$ is estimated at $1 \%$ for $t \simeq 1 / 10 s(\simeq 15 \%$ for $t \simeq 1 / 200 s)$. This confirms that the light flickering has a noticeable effect mainly for high ISO. In addition, a slight variability of the actual exposure can be measured. The small values of $\sigma_{\gamma}$ (yielding $E\left(v^{*}(t)\right)-1 \simeq E\left(v_{*}(t)\right) \simeq 0$, cf. (15-16)) also explain the poorer estimation of $\mu$ for low ISO. Moreover, the increasing $\sigma_{\gamma}$ makes the bias in method $\mathbf{4}$ to increase with ISO.

This paper discussed the effect of illumination flickering or slightly varying exposure on image noise measurement and proposed a practical algorithm, robust to rolling shutter effect. This approach could be useful for multi-image denoising [11]. 


\section{REFERENCES}

[1] G.E. Healey and R. Kondepudy, "Radiometric CCD camera calibration and noise estimation," IEEE Transactions on Pattern Analysis and Machine Intelligence, vol. 16, no. 3, pp. 267-276, 1994.

[2] J. Boulanger, C. Kervrann, P. Bouthemy, P. Elbau, J.-B. Sibarita, and J. Salamero, "Patch-based nonlocal functional for denoising fluorescence microscopy image sequences," IEEE Transaction on Medical Imaging, vol. 29, no. 2, pp. 442-454, 2010.

[3] H. Faraji and W.J. MacLean, "CCD noise removal in digital images," IEEE Transactions on Image Processing, vol. 15, no. 9, pp. 2676-2685, 2006.

[4] A. Foi, M. Trimeche, V. Katkovnik, and K. Egiazarian, "Practical Poissonian-Gaussian noise modeling and fitting for single-image raw-data," IEEE Transactions on Image Processing, vol. 17, no. 10, pp. 1737-1754, 2008.

[5] "Standard 1288, standard for characterization of image sensors and cameras, release 3.0," Tech. Rep., European Machine Vision Association (EMVA), 2010.

[6] F. Murthagh, J.L. Starck, and A. Bijaoui, "Image restoration with noise suppression using a multiresolution support," Astronomy and astrophysics, vol. 112, pp. 179-189, 1995.

[7] S. Delpretti, F. Luisier, S. Ramani, T. Blu, and M. Unser, "Multiframe sure-let denoising of timelapse fluorescence microscopy images," in Proceedings of the IEEE International Symposium on Biomedical Imaging (ISBI), 2008, pp. 149-152.

[8] S. Ramani, C. Vonesch, and M. Unser, "Deconvolution of $3 \mathrm{~d}$ fluorescence micrographs with automatic risk minimization," in Proceedings of the IEEE International Symposium on Biomedical Imaging (ISBI), 2008, pp. 732-735.

[9] C. Liu, R. Szeliski, S.B. Kang, C.L. Zitnick, and W.T. Freeman, "Automatic estimation and removal of noise from a single image," IEEE Transactions on Pattern Analysis and Machine Intelligence, vol. 30, no. 2, pp. 299-314, 2008.

[10] A. Foi, S. Alenius, V. Katvonik, and K. Egiazarian, "Noise measurement for raw-data of digital imaging sensors by automatic segmentation of non-uniform targets," IEEE Sensors Journal, vol. 7, no. 10, pp. 14561461, 2007.

[11] T. Buades, Y. Lou, J.-M. Morel, and Z. Tang, "A note on multi-image denoising," in Proceeding of the International Workshop on Local and Non-Local Approximation in Image Processing, Tuusalu, Finland, 2009.
[12] J. Delon, "Midway image equalization," Journal of Mathematical Imaging and Vision, vol. 21, no. 2, pp. 119-134, 2004.

[13] F.R. Boddekke, Quantitative fluorescence microscopy, Ph.D. thesis, Technische Universiteit Delft, 1999.

[14] P. Roudot, C. Kervrann, F. Waharte, and J. Boulanger, "Lifetime map reconstruction in frequency-domain fluorescence lifetime imaging microscopy," in Proceedings of the International Conference on Image Processing (ICIP), Orlando, Florida, USA, 2012, pp. 2537-2540.

[15] F. Sur and M. Grédiac, "Sensor noise modeling by stacking pseudo-periodic grid images affected by vibrations," IEEE Signal Processing Letters, vol. 21, no. 4, pp. 432-436, 2014.

[16] B. Waegli, "Investigations into the noise characteristics of digitized aerial images," International Archives for Photogrammetry and Remote Sensing, vol. XXXII/2, pp. 341-348, 1998.

[17] J. Delon and A. Desolneux, "Stabilization of flickerlike effects in image sequences through local contrast correction," SIAM Journal on Imaging Sciences, vol. 3, no. 4, pp. 703-734, 2010.

[18] A. Buades, B. Coll, and J.-M. Morel, "A review of image denoising algorithms, with a new one," SIAM Journal on Multiscale Modeling and Simulation, vol. 4, no. 2, pp. 490-530, 2005. 